\title{
Computing Genetic Similarity Coefficients from RAPD Data: The Effects of PCR Artifacts
}

\author{
Warren F. Lamboy \\ Department of Horticultural Sciences and U.S. Department of Agriculture, Agricultural Research Service (USDA-ARS), Plant \\ Genetic Resources Unit, Cornell University, Geneva, New York 14456-0462
}

Random amplified polymorphic DNA (RAPD) markers have been used for many types of genetic analyses, including genome mapping, genotype fingerprinting, phylogeny reconstruction, and measuring genetic similarities. They suffer from one potential limitation, however, because the PCR that is used to produce informative amplification products often produces artifactual products as well. Optimization of PCR protocols to eliminate artifactual bands completely is often too costly or too timeconsuming to be practical. Other methods for handling RAPD artifacts, such as deleting inconsistent or faint bands or using only those bands that are reproducible, introduce false negatives into the data. Simply ignoring artifacts and using all bands introduces false positives. When RAPD data are used to compute genetic similarity coefficients, such artifacts can cause significant bias in the estimation. The three coefficients most widely used with RAPD data, the simple matching coefficient, Jaccard's coefficient and Nei and Li's coefficient, differ in the amount of bias produced by a given level of artifactual bands. The simple matching coefficient and Nei and Li's coefficient always exhibit less percent bias than Jaccard's coefficient. For closely related organisms, Nei and Li's coefficient displays less percent bias than the simple matching coefficient. If new DNA samples possessing RAPD markers not present in the previously analyzed samples are added to a study, values of the simple matching coefficient will need to be computed for all samples, not just the new ones. Jaccard's and Nei and Li's coefficients, however, will not need to be recomputed. Furthermore, only Nel and Li's coefficient has a direct biological meaning (it is an estimate of the expected proportion of amplified fragments shared by two samples because they were inherited from a common ancestor). On the basis of these results, Nei and Li's coefficient is recommended for routine computation of genetic similarities using RAPD data, particularly if PCR artifacts are present.

D

Landom amplified polymorphic DNA (RAPD) markers ${ }^{(1,2)}$ are well-established genetic tools. They have been used for genomic mapping and linkage analysis, ${ }^{(3-6)}$ phylogeny reconstruction, ${ }^{(7,8)}$ genotype fingerprinting and identification, ${ }^{(9-14)}$ and determining genetic relationships, similarities, and genetic variation. (15-17)

RAPD bands are produced by PCR, using a single random primer that amplifies segments of DNA flanked by two primer-binding regions that theoretically are exactly complementary to the primer. The primer-binding sites must be close enough that amplification proceeds over the entire DNA segment spanning them. Because of base pair mismatch, though, a single base change in the genomic DNA can prevent amplification. ${ }^{(2)}$ Most often, polymorphisms between different DNA samples occur when a segment that is amplified in a sample whose primer-binding site is exactly complementary to the primer is not amplified in another sample whose priming site is not an exact complement to the primer. ${ }^{(2,18)}$ Polymorphisms may also result from deletions within a priming site, insertions that separate priming sites by too great a distance to support amplification, or insertions that change the size of the DNA segment without affecting amplification. (2) Segments that can be amplified in one genetic background but not in another, ${ }^{(19)}$ polymerase slippage during replication, nontemplate-directed addition of nucleotides by Taq polymerase, or the amplification of in vitro recombinants ${ }^{(20)}$ also result in the production of polymorphisms. Even changes in PCR parameters, such as primer/template ratios, annealing temperature, and $\mathrm{Mg}^{2+}$ concentration $^{(21)}$ can cause polymorphisms, because bands appear or disappear when amplification conditions are altered. DNA concentration can have a strong effect if there is contaminating RNA that is mistakenly taken for DNA, ${ }^{(21)}$ and DNA containing significant amounts of polysaccharides, phenolics, or other impurities can cause polymorphisms. ${ }^{(22)}$ Thermocyclers themselves may be responsible for variability in RAPD banding patterns. ${ }^{(23)}$

Whatever their source, artifactual RAPD bands belong to one of two types: bands that appear in a lane but should not (false positives), or bands that do not appear in a lane but should (false negatives). This designation is arbitrary to a certain degree. For example, if the offspring of two known parents exhibits a RAPD band not shown in either parent, 
in the offspring the band could be a false positive while in the parents the lack of the band could be a false negative (barring mutation, crossing-over, etc., involving the amplified site). Consequently, in the absence of specific information about the DNA sequences that resulted in the amplified fragments, presence of bands in some samples and absence in others could represent either false positives or false negatives.

Workers using RAPDs are well aware of the possibility of artifactual bands and of their possible effects on subsequent

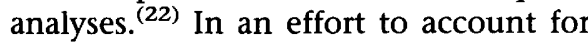
artifacts and to reduce their effects on analyses, a number of different approaches have been adopted. These include discarding faint or inconsistent bands, ${ }^{(4-6,8,10,11,14,15,17)}$ carrying out replicate runs and analyzing only those bands that were reproducible, ${ }^{(3,9)}$ or using all bands and accepting a certain level of error. ${ }^{(7)}$

Each of the methods of dealing with artifactual bands has its advantages and disadvantages. Discarding faint or inconsistent bands results in loss of information and increases the number of false negatives (bands that should have been present but were not, because they were discarded by the experimenter). Replicating runs and using only those bands that appear in all replicates is an improvement upon ignoring bands but requires at least double the number of amplification runs. Moreover, to be true replicates in the statistical sense requires that all possible sources of error be accounted for by the replicates. In practice this means that the entire procedure from DNA extraction, to amplification, to electrophoresis must be performed independently for each replicate. Use of all amplification products, whether faint or strong, consistent or not, does not result in any loss of informative data, and no additional false negatives are produced, but it does retain all false positives. None of these methods of handling artifactual bands completely solves the problem of artifactual bands.

This paper focuses on the use of RAPDs for the computation of genetic similarity coefficients when optimization of PCR protocols for eliminating artifacts is either not possible or not successful. Three different similarity measures are considered: (1) the simple matching coefficient, $S M C_{;}^{(7,24)}(2) \mathrm{Jac}-$ card's coefficient, $J ;^{(7,24-26)}$ and (3) Nei and Li's coefficient, $N L .^{(10,16,27-29)}$ These coefficients are examined in terms of the true value that they are designed to estimate using RAPD data containing no artifactual bands and in terms of the estimated value that they actually measure when artifactual bands (either false positives or false negatives or both) are present. Using those results, properties of and relationships among the coefficients are determined. The amount of bias (difference between the estimated value and the true value) in the estimated coefficients is examined for several levels of artifactual bands. On the basis of these results, a recommendation is made as to which coefficient is the best for routine use with RAPD data.

\section{MATERIALS AND METHODS}

The three similarity coefficients can be defined in terms of the sets of RAPD bands present in two amplified DNA samples, for example, sample i and sample j. $S M C$ is the sum of the number of RAPD bands that are present in both samples plus the number of RAPD bands absent in both samples, divided by the total number of different possible bands in the study. $J$ is the number of bands present in both samples, divided by the quantity the number of bands present in sample $i$ plus the number of bands present in sample $j$ minus the number of bands present in both samples. Finally, $N L$ is the number of RAPD bands present in both samples, divided by the average number of bands present in sample $i$ and sample $j$. Although these definitions can be and are used for computing the coefficients from raw data, they do not allow for convenient comparison between the coefficients. For that purpose, the following notation is needed.

In any study in which RAPD data will be used to compute pairwise similarities, there will be at least two and usually many different DNA samples for which similarities will be computed. Let $n$ represent the number of different samples. Then, the set of bands of interest are those bands that are present in one or more of the $n$ samples. To avoid excessive numbers of subscripts and to simplify the presentation, computation of genetic similarities using just two amplified DNA samples is considered. Because similarities are computed pairwise, the equations developed are completely gen- eral and can be applied to any pair of samples.

Any pair of samples (call them "sample i" and "sample $j$ ") will possess some of the same bands and lack some of the same bands. (A band not present in a particular pair of samples will be present, however, in at least one other sample under study.) Let the proportion of the total number of bands that are shared (either positive or negative) be $s$. Let $p$ represent the proportion of the shared bands that are shared because both samples possess the same bands. These bands are referred to as the shared positive bands. In general, the value for $p$ will be different for every pair of samples being compared. Then $1-p$ is the proportion of bands shared that both samples lack. These bands are referred to as the shared negative bands.

The proportion of bands that the samples do not share is $1-s$. Because these bands are unshared, some of them will be present only in sample $i$; the rest will be present only in sample j. Let $r$ be the proportion of unshared bands that are present in sample $\mathrm{i}$. Then $r$ also will represent the proportion of unshared bands that are absent in sample $j$. The proportion of unshared bands that are absent in sample $\mathrm{i}$, then, will be $1-r$, and the proportion of unshared bands that are present in sample $j$ is $1-r$.

Finally, let $f_{p}$ be the probability that a band that should not be present in a sample is present as an artifact; $f_{p}$ is the probability of a false positive. Let $f_{n}$ be the probability that a band that should be present in a sample is absent because of experimental error; $f_{n}$ is the probability of a false negative. In discussing computed similarity coefficients, the term "true value" means that value of any of the three coefficients that would result if there were no false positives and no false negatives. "Estimated value" or "estimate" means the value that results when the coefficients are computed using data containing false positives or false negatives or both.

\section{RESULTS AND DISCUSSION}

\section{The Three Coefficients in Terms of Numerical Values}

Using the notation introduced above, it is possible to write equations for the true values of each of the three coefficients as well as the equations for what the three 
coefficients actually estimate when false positives and false negatives are present.

The simple matching coefficient is the sum of the proportion of shared positive bands plus the proportion of shared negative bands. The true value for the simple matching coefficient is given by

$$
\begin{aligned}
S M C & =s p+(1-p) s \\
& =s
\end{aligned}
$$

Jaccard's coefficient considers only the proportion of positive bands shared by both samples and divides that by the proportion of positive bands in both samples plus the proportion of bands in sample i but not also in sample j plus the proportion of bands in sample $\mathrm{j}$ but not also in sample $i$. The true value for Jaccard's coefficient is

$$
\begin{aligned}
& J=\frac{s p}{s p+(1-s) r+(1-s)(1-r)} \\
& J=\frac{s p}{s p+(1-s)}
\end{aligned}
$$

Nei and Li's coefficient is the only one of the three that has a straightforward biological interpretation. It measures the proportion of fragments that two samples share because they have been inherited from a common ancestor. The coefficient was originally derived for restriction fragment data, ${ }^{(27)}$ but the formula applies equally well to amplified fragments. It is computed as the proportion of positive bands shared by both samples, divided by the average of the proportion of bands present in sample i and sample $\mathrm{j}$. The true value for Nei and Li's coefficient can be written as

$$
\begin{aligned}
N L= & s p /[s p+(1-s) r+s p \\
& +(1-s)(1-r)] / 2 \\
= & \frac{2 s p}{2 s p+(1-s)}
\end{aligned}
$$

Note that the parameter $r$ does not appear in any of these equations. Thus, it does not matter which of the unshared bands belong to which sample; all that matters is that they are unshared.

Using the above equations, it can be shown that for a specific pair of samples the following relationships hold among the three coefficients.

$\begin{aligned} S M C & \geqslant J \text { for } s \in[0,1], p \in[0,1] \\ N L & \geqslant J \text { for } s \in[0,1], p \in[0,1] \\ S M C & >N L \text { for } s \in[0,1], p<1 / 2 \\ S M C & =N L \text { for } s \in[0,1], p=1 / 2 \\ S M C & <N L \text { for } s \in[0,1], p>1 / 2\end{aligned}$

Furthermore, $S M C, I$, and $N L$ are monotonic with respect to one another, that is, if the data change in a way that increases the value of one of the coefficients, it will increase the value of all of them. Similarly if one of the coefficients decreases in value, all of them will.

From the definition of the coefficients, it can be shown that if additional DNA samples are added to a study after similarities have already been computed for the earlier samples, and one or more of the new DNA samples possess bands that were not possessed by any of the samples analyzed previously, values of $S M C$ need to be computed (or recomputed) for all samples, not just for the new samples, because the value of $S M C$ for each pair of samples depends on the total number of bands that could possibly be present in a sample. Values of $J$ and $N L$, on the other hand, do not need to be recomputed for samples studied previously, as these coefficients depend only on the positive bands in the two samples being compared, not on bands that are present in other samples. For this reason, in a study where data are accumulated for different samples over a long period of time and the similarity coefficients are computed as data are collected, $J$ and $N L$ have some advantage over SMC.

\section{Quantities Estimated by the Coefficients When False-positive and False-negative Bands are Present}

The true values of the coefficients in terms of the parameters $s$ and $p$ are shown in equations 1,2 , and 3 . Now it can be asked, When the three coefficients are computed according to these equations and false positives or false negatives or both are present in the data, what will the quantities computed actually be measuring? In general, of course, the values estimated will differ from the true values of the coefficients. The expected difference between the estimated value and the true value is called the bias. If the bias is positive, it indicates that the estimate is larger than the true value, and if the bias is negative, it indicates that the estimate is smaller than the true value.

To prepare explicit formulae for what is being computed using equations 1,2 , and 3 when there are artifactual bands, two intermediate quantities need to be determined. The first of these is the proportion of positive bands that are shared by the two samples when false positives and false negatives are present. Let this value be indicated by the symbol SP (for shared positive bands).

$$
\begin{aligned}
S P= & s p\left(1-f_{n}\right)^{2}+s(1-p) f_{p}^{2} \\
& +(1-s)\left(1-f_{n}\right) f_{p}
\end{aligned}
$$

The terms on the right-hand side of the equal sign in this equation were obtained as follows. The left-most term is the proportion of bands truly shared by both samples, neither of which became false negatives (caused by experimental error). The middle term is the proportion of bands truly not possessed by either sample, both of which became false positives (because of experimental error). The right-most term is the proportion of bands, one of which is truly a positive band that did not become a false negative and the other of which is truly a negative band that did become a false positive.

The second quantity needed is the proportion of shared negative bands, indicated by $S N$, and it is computed as

$$
\begin{aligned}
S N= & s p f_{n}^{2}+s(1-p)\left(1-f_{p}\right)^{2} \\
& +(1-s)\left(1-\mathrm{f}_{\mathrm{p}}\right) f_{n}
\end{aligned}
$$

The terms in this equation can derived by arguments analogous to those for equation 5. Having computed $S P$ and $S N$ it is easy to state what the coefficients are estimating when artifactual bands are present

$$
e s t(S M C)=S P+S N
$$

$\operatorname{est}(J)=[(S P+S N) p] /[(S P+S N) p+1$ $-(S P+S N)]$ 
$\operatorname{est}(N L)=[2(S P+S N) p] /[2(S P+S N) p$

$+1-(S P+S N)]$

Written out in terms of $s, p, r, f_{n}$, and $f_{p}$, equations 7,8 , and 9 are

$$
\begin{aligned}
\operatorname{est}(S M C)= & f_{n}+f_{p}-2 f_{n} f_{p} \\
& +\left(-1+f_{n}+f_{p}\right) \\
& \left(-1+2 f_{p}+2 p f_{n}-2 p f_{p}\right) s
\end{aligned}
$$

$$
\begin{aligned}
\operatorname{est}(D)= & {\left[p\left(f_{n}+f_{p}-2 f_{n} f_{p}\right)+s p(-1\right.} \\
& \left.+f_{n}+f_{p}\right)\left(-1+2 f_{p}+2 p f_{n}\right. \\
& \left.\left.-2 p f_{p}\right)\right] /\left[1-f_{n}-f_{p}+2 f_{n} f_{p}\right. \\
& +p\left(f_{n}+f_{p}-2 f_{n} f_{p}\right)+s(-1 \\
& \left.+f_{n}+f_{p}\right)(-1+p)\left(-1+2 f_{p}\right. \\
& \left.\left.+2 p f_{n}-2 p f_{p}\right)\right]
\end{aligned}
$$

$$
\begin{aligned}
\operatorname{est}(N L)= & {\left[2 p\left(f_{n}+f_{p}-2 f_{n} f_{p}\right)\right.} \\
& +2 s p\left(-1+f_{n}+f_{p}\right)(-1 \\
& \left.\left.+2 f_{p}+2 p f_{n}-2 p f_{p}\right)\right] / \\
& {\left[1-f_{n}-f_{p}+2 f_{n} f_{p}+2 p\left(f_{n}\right.\right.} \\
& \left.+f_{p}-2 f_{n} f_{p}\right)+s\left(-1+f_{n}+\right. \\
& \left.f_{p}\right)(-1+2 p)\left(-1+2 f_{p}+\right. \\
& \left.\left.2 p f_{n}-2 p f_{p}\right)\right]
\end{aligned}
$$

Note that the parameter $r$ does not appear in equation 10,11 , or 12 , just as it did not appear in 1,2 , or 3 . An examination of the equations shows that depending on the values of $s, p, f_{p}$, and $f_{n}$, the estimates may be either greater than (positive bias) or less than (negative bias) the true values. For any of the three coefficients, given values for $s, p, f_{p}$, and $f_{n}$, one can compute the bias by subtracting the true value from the expected value of the estimate.

\section{Bias in the Computed Values of the Coefficients}

Table 1 shows the true value, the estimate, the bias, and the bias as a percent of the true value for all three coefficients for a variety of values of $s(0.1,0.3,0.5$, 0.7 , and 0.9$), p(0.3,0.5,0.7), f_{p}(0.00$, $0.05,0.10)$, and $f_{n}(0.00,0.05,0.10)$. The values selected for $s$ were chosen to span the range of possible similarities, the values for $p$ were chosen because they are typical of what is found in real data, and the values of $f_{p}$ and $f_{n}$ were selected because $10 \%$ false positives or $10 \%$ false negatives seemed to be reasonable upper limits on the percent artifacts in an experimental study. ${ }^{(22)}$ It is possible, how- ever, that rates of artifacts could exceed these levels, even with PCR protocol optimization. Under such circumstances, genetic similarity coefficients should probably be corrected for the bias caused by these artifacts, using a method described in a companion manuscript. ${ }^{(30)}$

Bias calculations show that as expected, there is no bias if there are no false positives and no false negatives. In addition, all three coefficients exhibit no bias if $s=0.5$, and $f_{p}=f_{n}$.

The percent bias for $S M C$ and $N L$ is always less than or equal to the percent bias for $J$, for all values of the parameters tested. The percent bias is smaller for $S M C$ than it is for $N L$ if $p<0.5$, is equal to the percent bias for $N L$ if $p=0.5$, and is greater than the percent bias for $N L$ if $p>0.5$. A specific level of false negatives causes greater percent bias than the same level of false positives for all three coefficients if $p<0.5$. If $p>0.5$, then false positives cause greater percent bias than false negatives. The effects of false positives and false negatives are the same if $p=0.5$.

If $s<0.5$ the percent bias is positive for all values of false positives and false negatives examined; if $s>0.5$, the percent bias is negative. It is worth considering why this is the case taking the $S M C$ as an example. If $s<0.5$, then more than onehalf of the possible bands are present in one sample and absent in the other. If there are false positives, then the proportion of matching negative bands that are made nonmatching (as one is a false positive) are less than the proportion of nonmatching bands that are made matching because a previously negative band was made positive (and so matches its positive counterpart). If there are false negatives, then again because over one half of the possible bands did not match, the previously positive bands that are false negatives now match their negative counterparts. There is a greater proportion of these than there is of positive matches that are broken up. A similar line of argument explains why the bias is always negative if $s>0.5$. If $s=0.5$, the bias is sometimes positive and sometimes negative, depending on the values of the other parameters.

For studies in which some level of bias can be tolerated, it can be asked which coefficient shows an acceptable level of bias, for example, less than $\pm 10 \%$ ? For the values of the parameters considered here, SMC and $N L$ show
$<10 \%$ bias $58.5 \%$ of the time, whereas $J$ exhibits $<10 \%$ bias $46.7 \%$ of the time. Furthermore, the percent bias displayed was less than $\pm 5 \% 41.5 \%$ of the time for $S M C$ and $N L$ and $33.3 \%$ of the time for $J$.

\section{CONCLUSIONS}

On the basis of these results, some recommendations can be made as to which coefficient should be used for assessing genetic similarity using RAPD data. First, if one is conducting a long-term study, in which similarities need to be computed as sample data arrive over a period of time, either the $J$ or $N L$ coefficient is preferable to $S M C$, as $S M C$ needs to be recomputed for all samples whenever a new RAPD band is added to the study. With $J$ and $N L$, only the similarities involving the new samples need to be computed.

The $N L$ coefficient is the only one of the three that has a biological meaning. It is an estimate of the expected proportion of shared amplified fragments. (27) The other two coefficients, although containing information on the similarity between samples, are related to biologically meaningful quantities only in unspecified ways.

The NL coefficient has yet another advantage. Because in closely related organisms (the kinds most often used in RAPD studies) most of the similarity between RAPD samples is caused by shared positive bands $(p>0.5), N L$ is preferred over $S M C$, because $N L$ displays less percent bias than either $S M C$ or $J$ in these circumstances.

Considering all of these factors, it is recommended that the $N L$ coefficient be used routinely for measuring similarities using RAPD data, unless specific circumstances or needs dictate the use of one of the other two coefficients. If one of the other two must be used, and recomputation of similarities with the arrival of new samples is not a consideration, SMC should be used rather than $J$, as it exhibits less percent bias. If recomputation is not feasible, then $J$ should be used.

If estimates of the percent of falsepositive and false-negative bands in the RAPD data are available (such as would be the case in theoretical studies or when replicate runs have been made), the equations developed here (1-3 and 1012) can be used to determine the actual bias by subtracting the true value from 
Table 1 Values of Similarity Coefficients and Estimates of Bias

\begin{tabular}{|c|c|c|c|c|c|c|c|c|c|c|c|c|c|c|c|}
\hline & & & & & & $S M C$ & & & & $J$ & & & & $N L$ & \\
\hline & Par & meters & & & & & & & & & & & & & \\
\hline$s$ & $p$ & $f_{p}$ & $f_{n}$ & value & valu & bias & $(\%$ & value & valu & bias & $(\%)$ & value & value & bias & $\begin{array}{l}\text { bras } \\
\text { (\%) }\end{array}$ \\
\hline 0.10 & 0.30 & 0.00 & 0.00 & 0.100 & 0.100 & 0.000 & 0.000 & 0.032 & 0.032 & 0.000 & 0.000 & 0.062 & 0.062 & .000 & 0.000 \\
\hline & 0.30 & 0.00 & 0.05 & 100 & 0.142 & 0.042 & 42.150 & 0.032 & 0.047 & 0.015 & 46.808 & 0.062 & 0.090 & 0.028 & 44.691 \\
\hline 0.10 & 0.30 & 0.00 & 0.10 & 0.100 & 0.185 & 0.085 & 84.600 & 0.032 & 0.064 & 0.031 & 97.154 & 0.062 & 0.120 & & \\
\hline 0.10 & 0.30 & 0.05 & 0.00 & 0.100 & 0.138 & 0.038 & 38.350 & 0.032 & 0.046 & 0.014 & 42.462 & 0.062 & 0.088 & 0.025 & 40.597 \\
\hline 0.10 & 0.30 & 0.05 & 0.05 & 0.100 & 0.176 & 0.076 & 76.000 & 0.032 & 0.060 & 0.028 & 86.679 & 0.062 & 0.114 & 0.051 & 81.756 \\
\hline 0.10 & 0.30 & 0.05 & 0.10 & 0.100 & 0.214 & 0.114 & 113.950 & 0.032 & 0.075 & 0.043 & 134.022 & 0.062 & 0.140 & 0.078 & 124.615 \\
\hline 0.10 & 0.30 & 0.10 & 0.00 & 0.100 & 0.177 & 0.077 & 77.400 & 0.032 & 0.061 & 0.029 & 88.374 & 0.062 & 0.115 & 0.052 & 83.312 \\
\hline 0.10 & 0.30 & 0.10 & 0.05 & 0.100 & 0.211 & 0.111 & 110.550 & 0.032 & 0.074 & 0.042 & 129.660 & 0.062 & 0.138 & 0.075 & 120.717 \\
\hline 0.10 & 0.30 & 0.10 & 0.10 & 0.100 & 0.244 & 0.144 & 144.000 & 0.032 & 0.088 & 0.056 & 173.661 & 0.062 & 0.162 & 0.100 & 159.574 \\
\hline 0.10 & 0.50 & 0.00 & 0.00 & 0.100 & 0.100 & 0.000 & 0.000 & 0.053 & 0.053 & 0.000 & 0.000 & 0.100 & 0.100 & 0.000 & 0.000 \\
\hline 0.10 & 0.50 & 0.00 & 0.05 & 0.100 & 0.140 & 0.040 & 40.250 & 0.053 & 0.075 & 0.023 & 43.285 & 0.100 & 0.140 & 0.040 & 40.250 \\
\hline 0.10 & 0.50 & 0.00 & 0.10 & 0.100 & 0.181 & 0.081 & 81.000 & 0.053 & 0.100 & 0.047 & 89.060 & 0.100 & 0.181 & 0.081 & 81.000 \\
\hline 0.10 & 0.50 & 0.05 & 0.00 & 0.100 & 0.140 & 0.040 & 40.250 & 0.053 & 0.075 & 0.023 & 43.285 & 0.100 & 0.140 & 0.040 & 40.250 \\
\hline 0.10 & 0.50 & 0.05 & 0.05 & 0.100 & 0.176 & 0.076 & 76.000 & 0.053 & 0.096 & 0.044 & 83.333 & 0.100 & 0.176 & 0.076 & 76.000 \\
\hline 0.10 & 0.50 & 0.05 & 0.10 & 0.100 & 0.212 & 0.112 & 112.250 & 0.053 & 0.119 & 0.066 & 125.577 & 0.100 & 0.212 & 0.112 & 112.250 \\
\hline 0.10 & 0.50 & 0.10 & 0.00 & 0.100 & 0.181 & 0.081 & 81.000 & 0.053 & 0.100 & 0.047 & 89.060 & 0.100 & 0.181 & 0.081 & 81.000 \\
\hline 0.10 & 0.50 & 0.10 & 0.05 & 0.100 & 0.212 & 0.112 & 112.250 & 0.053 & 0.119 & 0.066 & 125.577 & 0.100 & 0.212 & 0.112 & 112.250 \\
\hline 0.10 & 0.50 & 0.10 & 0.10 & 0.100 & 0.244 & 0.144 & 144.000 & 0.053 & 0.139 & 0.086 & 164.009 & 0.100 & 0.244 & 0.144 & 144.000 \\
\hline 0.10 & 0.70 & 0.00 & 0.00 & 0.100 & 0.100 & 0.000 & 0.000 & 0.072 & 0.072 & 0.000 & 0.000 & 0.135 & 0.135 & 0.000 & 0.000 \\
\hline 0.10 & 0.70 & 0.00 & 0.05 & 0.100 & 0.138 & 0.038 & 38.350 & 0.072 & 0.101 & 0.029 & 40.011 & 0.135 & 0.184 & 0.049 & 36.339 \\
\hline 0.10 & 0.70 & 0.00 & 0.10 & 0.100 & 0.177 & 0.077 & 77.400 & 0.072 & 0.131 & 0.059 & 81.751 & 0.135 & 0.232 & 0.097 & 72.272 \\
\hline 0.10 & 0.70 & 0.05 & 0.00 & 0.100 & 0.142 & 0.042 & 42.150 & .072 & 0.104 & 0.032 & 44.028 & 0.135 & 0.188 & 0.054 & 39.882 \\
\hline 0.10 & 0.70 & 0.05 & 0.05 & 0.100 & 0.176 & 0.076 & 76.000 & .072 & 0.130 & 0.058 & 80.236 & 0.135 & 0.230 & 0.096 & 71.001 \\
\hline 0.10 & 0.70 & 0.05 & 0.10 & 0.100 & 0.211 & 0.111 & 110.550 & .072 & 0.157 & 0.085 & 118.004 & 0.135 & 0.272 & 0.137 & 101.963 \\
\hline 0.10 & 0.70 & 0.10 & 0.00 & 0.100 & 0.185 & 0.085 & 84.600 & .072 & 0.137 & 0.065 & 89.560 & 0.135 & 0.241 & 0.106 & 78.783 \\
\hline 0.10 & 0.70 & 0.10 & 0.05 & 0.100 & 0.214 & 0.114 & 113.950 & .072 & 0.160 & 0.088 & 121.766 & 0.135 & 0.276 & 0.141 & 104.967 \\
\hline 0.10 & 0.70 & 0.10 & 0.10 & 0.100 & 0.244 & 0.144 & 144.000 & 0.072 & 0.184 & 0.112 & 155.373 & 0.135 & 0.311 & 0.177 & 131.195 \\
\hline 0.30 & 0.30 & 0.00 & 0.00 & 0.300 & 0.300 & 0.000 & 0.000 & .114 & 0.114 & 0.000 & 0.000 & 0.205 & 0.205 & 0.000 & 0.000 \\
\hline 0.30 & 0.30 & 0.00 & 0.05 & 0.300 & 0.326 & 0.026 & 8.817 & .114 & 0.127 & 0.013 & 11.428 & 0.205 & 0.225 & 0.021 & 10.141 \\
\hline 0.30 & 0.30 & 0.00 & 0.10 & 0.300 & 0.354 & 0.054 & 17.933 & .114 & 0.141 & 0.0 & 23.837 & 0.205 & 0.247 & 0.043 & 20.890 \\
\hline 0.30 & 0.30 & 0.05 & 0.00 & 0.300 & 0.315 & 0.015 & 5.017 & 0.114 & 0.121 & 0.007 & 6.436 & 0.205 & 0.216 & 0.012 & 5.740 \\
\hline 0.30 & 0.30 & 0.05 & 0.05 & 0.300 & 0.338 & 0.038 & 12.667 & .114 & 0.133 & 0.019 & 16.592 & 0.205 & 0.235 & 0.030 & 14.647 \\
\hline 0.30 & 0.30 & 0.05 & 0.10 & 0.300 & 0.362 & 0.062 & 20.617 & 0.114 & 0.145 & 0.031 & 27.610 & 0.205 & 0.254 & 0.049 & 24.106 \\
\hline 0.30 & 0.30 & 0.10 & 0.00 & 0.300 & 0.332 & 0.032 & 10.733 & .114 & 0.130 & 0.016 & 13.986 & 0.205 & 0.230 & 0.025 & 12.378 \\
\hline 0.30 & 0.30 & 0.10 & 0.05 & 0.300 & 0.352 & 0.052 & 17.217 & 0.114 & 0.140 & 0.026 & 22.838 & 0.205 & 0.246 & 0.041 & 20.035 \\
\hline 0.30 & 0.30 & 0.10 & 0.10 & 0.300 & 0.372 & 0.072 & 24.000 & .114 & 0.151 & 0.037 & 32.450 & 0.205 & 0.262 & 0.058 & 28.195 \\
\hline 0.30 & 0.50 & 0.00 & 0.00 & 0.300 & 0.300 & 0.000 & 0.000 & .176 & 0.176 & 0.000 & 0.000 & 0.300 & 0.300 & 0.000 & 0.000 \\
\hline 0.30 & 0.50 & 0.00 & 0.05 & 0.300 & 0.321 & 0.021 & 17 & .176 & 0.191 & 0.015 & 38 & 0.300 & 0.321 & 0. & 6.917 \\
\hline 0.30 & 0.50 & 0.00 & 0.10 & 0.300 & 0.343 & 0.043 & 14.333 & 0.176 & 0.207 & 0.031 & 17.300 & 0.300 & 0.343 & 0.043 & 14.333 \\
\hline 0.30 & 0.50 & 0.05 & 0.00 & 0.300 & 0.321 & 0.021 & 6.917 & .176 & 0.191 & 0.015 & 8.238 & 0.300 & 0.321 & 0.021 & 6.917 \\
\hline 0.30 & 0.50 & 0.05 & 0.05 & 0.300 & 0.338 & 0.038 & 12.667 & 0.176 & 0.203 & 0.027 & 15.243 & 0.300 & 0.338 & 0.038 & 67 \\
\hline 0.30 & 0.50 & 0.05 & 0.10 & 0.300 & 0.357 & 0.057 & 1 & .176 & 0.217 & 0.0 & 3 & 0.300 & 0.357 & 0. & 18.917 \\
\hline 0.30 & 0.50 & 0.10 & 0.00 & 0.300 & 0.343 & 0.043 & 14.333 & 0.176 & 0.207 & 0.031 & 17.300 & 0.300 & 0.343 & 43 & 33 \\
\hline 0.30 & 0.50 & 0.10 & 0.05 & 0.300 & 0.357 & 0.057 & 18.917 & 0.176 & 0.217 & 0.041 & 23.023 & 0.300 & 0.357 & 0.057 & 18.917 \\
\hline 0.30 & 0.50 & 0.10 & 0.10 & 0.300 & 0.372 & 0.072 & 24.000 & 0.176 & 0.2 & 0.052 & 29.484 & 0.300 & .372 & 0. & 24.000 \\
\hline 0.30 & 0.70 & 0.00 & 0.00 & 0.300 & 0.300 & 0.000 & 0.000 & 0.231 & 0.231 & 0.000 & 0.000 & 0.375 & 0.375 & 0. & 0.000 \\
\hline 0.30 & 0.70 & 0.00 & 0.05 & 0.300 & 0.315 & 0.015 & 5.017 & 0.231 & 0.244 & 0.013 & 5.540 & 0.375 & . 392 & 0. & 4.455 \\
\hline 0.30 & 0.70 & 0.00 & 0.10 & 0.300 & 0.332 & 0.032 & 10.733 & 0.231 & 0.258 & 0.028 & 11.921 & 0.375 & 0.411 & 0.0 & 9.474 \\
\hline 0.30 & 0.70 & 0.05 & 0.00 & 0.300 & 0.326 & 0.026 & 8.817 & 31 & 0 & 0.023 & 9.774 & 75 & .404 & 0. & 7.798 \\
\hline 0.30 & 0.70 & 0.05 & 0.05 & 0.300 & 0.338 & 0.038 & 12.667 & 0.231 & 0.2 & 0.033 & 14.096 & 0.375 & 0.417 & 0.042 & 11.158 \\
\hline 0.30 & 0.70 & 0.05 & 0.10 & 0.300 & 0.352 & 0.052 & 17.2 & 0.2 & 0.275 & 0.044 & & .375 & 0.432 & 0. & 15.094 \\
\hline 0.30 & 0.70 & 0.10 & 0.00 & 0.300 & 0.354 & 0.054 & 17.9 & 0.231 & 0.277 & 0.046 & 20.063 & 0.375 & 0.434 & 0. & 15.710 \\
\hline 0.30 & 0.70 & 0.10 & 0.05 & 0.300 & & 0.062 & & 0.2 & 0.284 & & & 0.375 & 0.443 & 0. & 18.010 \\
\hline 0.30 & 0.70 & 0.10 & 0.10 & 0.300 & 0.372 & 0.072 & 24.0 & 0.2 & 0.293 & 0. & 27 & 0.375 & 0.453 & 0.078 & 20.891 \\
\hline 0.50 & 0.30 & 0.00 & 0.00 & 0.500 & 0.500 & 0.000 & 0.0 & 0 & 0.231 & 0. & & 0.375 & 0.375 & 0.000 & 0.000 \\
\hline 0.50 & 0.30 & 0.00 & 0.05 & 0.500 & 0.511 & 0.011 & 2.1 & & 0.238 & 0. & 46 & 0.375 & 0.385 & & 702 \\
\hline 0.50 & 0.30 & 0.00 & 0.10 & 0.500 & 0.523 & 0.023 & 4.6 & & 0.2 & 0. & 7. & 0.375 & 0.397 & 0.022 & 5.817 \\
\hline 0.50 & 0.30 & 0.05 & 0.00 & 0.500 & 0.492 & -0.008 & -1.650 & 0.231 & 0.225 & -0.006 & -2.516 & 0.375 & 0.367 & -0.008 & -2.054 \\
\hline 0.50 & 0.30 & 0.05 & 0.05 & 0.5 & 0.500 & 000 & 0.0 & 1 & 0.231 & 0.000 & 0.000 & 0.375 & 0.375 & 0.000 & 0.000 \\
\hline 0.50 & 0.30 & 0.05 & 0.10 & 0.500 & 0.510 & 0.010 & 1.95 & 0.231 & 0.238 & 0.007 & 3.032 & 0.375 & 0.384 & & \\
\hline 0.50 & 0.30 & 0.10 & 0.00 & 0.500 & 0.487 & -0.013 & -2. & 0.231 & 0.222 & -0.009 & -3.945 & 0.375 & 0.363 & -0.012 & -3 \\
\hline 0.50 & 0.30 & 0.10 & 0.05 & 0.500 & 0.493 & -0.007 & -1.450 & 0.231 & 0.226 & -0.005 & -2.213 & 0.375 & 0.368 & -0.007 & -1.806 \\
\hline 0.50 & 0.30 & 0.10 & 0.10 & 0.500 & 0.500 & 0.000 & & & & & (200 & 0.375 & 0.375 & 0.000 & 0.000 \\
\hline 0.50 & 0.50 & 0.00 & 0.00 & 0.500 & 0.500 & 0.000 & 0.000 & 0.333 & 0.33 & 0. & 0.000 & 0.500 & & & \\
\hline 0.50 & 0.50 & 0.00 & 0.05 & & 0.501 & 0.001 & 0.2 & & & 0.001 & & 0.500 & & & 0.250 \\
\hline 0.50 & 0.50 & 0.00 & 0.10 & 0.500 & 0.505 & 0.005 & 1.000 & 0.333 & 0.338 & 0.004 & 1.338 & 0.500 & 0.505 & 0.005 & 1.000 \\
\hline 0.50 & 0.50 & 0.05 & 0.00 & 0.500 & 0.501 & 0.001 & & & & 0.001 & 0.334 & 0.500 & 0.501 & 0.001 & 0.250 \\
\hline 0.50 & 0.50 & 0.05 & 0.05 & 0.500 & 0.500 & 0.000 & 0.000 & 0.333 & 0.333 & 0.000 & 0.000 & & & & 0.000 \\
\hline 0.50 & 0.50 & 0.05 & 0.10 & 0.500 & 0.501 & 0.001 & & & & 0.001 & & 0.500 & 0.501 & $0 . c$ & 0.250 \\
\hline 0.50 & 0.50 & 0.10 & 0.00 & 0.500 & 0.505 & 0.005 & 1.000 & 0.333 & 0.338 & 0.004 & 1.338 & 0.500 & 0.505 & 0.005 & 1.000 \\
\hline 0.50 & 0.50 & 0.10 & 0.05 & 0.500 & 0.501 & 0.001 & 0.250 & 0.333 & 0.334 & 0.001 & 0.334 & 0.500 & 0.501 & 0.001 & 0.250 \\
\hline 0.50 & 0.50 & 0.10 & 0.10 & 0.500 & 0.500 & 0.000 & 0.000 & 0.333 & 0.333 & 0.000 & 0.000 & Do & 0.500 & 0.000 & 0.000 \\
\hline 0.50 & 0.70 & 0.00 & 0.00 & 0.500 & 0.500 & 0.000 & & & 0.412 & & 0.000 & 0.583 & 0.583 & 0.000 & 0.000 \\
\hline 0.50 & 0.70 & 0.00 & 0.05 & 0.500 & 0.492 & -0.008 & -1.650 & 0.412 & 0.404 & -0.008 & 36 & 0.583 & 0.575 & -0.008 & -1.379 \\
\hline 0.50 & 0.70 & 0.00 & 0.10 & 0.500 & 0.487 & -0.013 & 2.600 & 0.412 & 0.399 & -0.013 & -3.045 & 0.583 & 0.571 & -0.013 & -2.176 \\
\hline
\end{tabular}


Table 1 (Continued)

\begin{tabular}{|c|c|c|c|c|c|c|c|c|c|c|c|c|c|c|c|}
\hline & & & & & & $S M C$ & & & & $J$ & & & & $N L$ & \\
\hline & $\mathrm{Pa}$ & eters & & & & & & & & & & & & & \\
\hline$S$ & $p$ & $f_{p}$ & $f_{n}$ & $\begin{array}{l}\text { true } \\
\text { value }\end{array}$ & value & bias & $\begin{array}{l}\text { bias } \\
(\%)\end{array}$ & $\begin{array}{l}\text { true } \\
\text { value }\end{array}$ & $\begin{array}{l}\text { mated } \\
\text { value }\end{array}$ & bias & $\begin{array}{l}\text { bias } \\
(\%)\end{array}$ & $\begin{array}{l}\text { true } \\
\text { value }\end{array}$ & value & bias & $\begin{array}{l}\text { bias } \\
(\%)\end{array}$ \\
\hline $\begin{array}{l}0.50 \\
0.50 \\
0.50 \\
0.50 \\
0.50 \\
0.50\end{array}$ & $\begin{array}{l}0.70 \\
0.70 \\
0.70 \\
0.70 \\
0.70 \\
0.70\end{array}$ & $\begin{array}{l}0.05 \\
0.05 \\
0.05 \\
0.10 \\
0.10 \\
0.10\end{array}$ & $\begin{array}{l}0.00 \\
0.05 \\
0.10 \\
0.00 \\
0.05 \\
0.10\end{array}$ & $\begin{array}{l}500 \\
500 \\
500\end{array}$ & $\begin{array}{l}0.511 \\
0.500 \\
0.493 \\
0.523 \\
0.510 \\
0.500\end{array}$ & $\begin{array}{r}0.011 \\
0.000 \\
-0.007 \\
0.023 \\
0.010 \\
0.000\end{array}$ & $\begin{array}{r}2.150 \\
0.000 \\
-1.450 \\
4.600 \\
1.950 \\
0.000\end{array}$ & $\begin{array}{l}0.412 \\
0.412 \\
0.412 \\
0.412 \\
0.412 \\
0.412\end{array}$ & $\begin{array}{l}.422 \\
.412 \\
.405 \\
.434 \\
.421 \\
.412\end{array}$ & $\begin{array}{r}0.010 \\
0.000 \\
-0.007 \\
0.022 \\
0.009 \\
0.000\end{array}$ & $\begin{array}{r}2.539 \\
0.000 \\
-1.702 \\
5.456 \\
2.302 \\
0.000\end{array}$ & $\begin{array}{l}.583 \\
.583 \\
.583 \\
.583 \\
.583 \\
.583\end{array}$ & $\begin{array}{l}0.594 \\
0.583 \\
0.576 \\
0.506 \\
0.593 \\
0.583\end{array}$ & $\begin{array}{r}0.010 \\
0.000 \\
-0.007 \\
0.022 \\
0.009 \\
0.000\end{array}$ & $\begin{array}{r}1.785 \\
0.000 \\
-1.211 \\
3.804 \\
1.620 \\
0.000\end{array}$ \\
\hline 0.70 & 0.30 & 0.00 & 0.00 & 0.700 & 0.700 & 0.000 & 0.000 & 0.412 & 0.412 & 0.000 & 0.000 & .583 & 0.583 & 0.000 & 0.000 \\
\hline $\begin{array}{l}0.70 \\
0.70\end{array}$ & $\begin{array}{l}0.30 \\
0.30\end{array}$ & $\begin{array}{l}0.00 \\
0.00\end{array}$ & $\begin{array}{l}0.05 \\
0.10\end{array}$ & & 0.695 & -0.005 & -0.707 & $\begin{array}{l}0.412 \\
0.412\end{array}$ & $\begin{array}{l}0.406 \\
0.403\end{array}$ & $\begin{array}{l}-0.006 \\
-0.009\end{array}$ & $\begin{array}{l}-1.377 \\
-2.162\end{array}$ & & $\begin{array}{l}0.578 \\
0.574\end{array}$ & $\begin{array}{l}-0 . \\
-0 .\end{array}$ & $\begin{array}{l}-0.979 \\
-1.541\end{array}$ \\
\hline 0.70 & 0.30 & .05 & 0.00 & & 0.668 & -0.032 & -4.507 & 0.412 & $\begin{array}{l}.403 \\
.377\end{array}$ & -0.035 & -8.471 & .583 & 0.547 & -0 . & -6.152 \\
\hline 0.70 & 0.30 & 0.05 & 0.05 & 100 & 0.662 & -0.038 & -5.429 & 0.412 & .370 & -0.042 & -10.117 & .583 & 0.540 & -0.043 & -7.384 \\
\hline 0.70 & 0.30 & .05 & 0.10 & & 0.658 & -0.042 & -6050 & 0.412 & .366 & -0.046 & & & 0.535 & -0.0 & -8.210 \\
\hline 0.70 & 0.30 & 0.10 & 0.00 & 10 & 0.642 & -0.058 & -8.314 & 0.412 & .350 & -0.062 & 15.09 & & 0.518 & -0.065 & -11.186 \\
\hline 0.70 & 0.30 & 10 & 0.05 & & .634 & -0.066 & -9.450 & 412 & .342 & -0.070 & 16.98 & & 0.509 & -0 . & -12.660 \\
\hline 0.70 & 0.30 & .10 & 0.10 & & 0.628 & -0.072 & 0.286 & 0.412 & .336 & -0.07 & 8,354 & & 0.503 & -0. & -13.736 \\
\hline 0.70 & 0.50 & .00 & 0.0 & & .700 & 0.000 & 0.1 & 0.53 & .538 & 0.00 & & & & & 0.000 \\
\hline 0.70 & 0.50 & .00 & 0.05 & & 0.682 & -0.0 & -2.6 & & .517 & -0.02 & -3 . & & 0.682 & -0 . & -2.607 \\
\hline 0.70 & 0.50 & 0.00 & 0.1 & & 0.667 & -0.0 & -4.7 & & 0 & -0.0 & -7 . & & 0.667 & -0 . & \\
\hline 0.70 & 0.50 & 0.05 & 0.00 & & 0.682 & -0.01 & -2.6 & & .517 & -0.02 & -3 & & 0.682 & -0. & -2.607 \\
\hline 0.70 & 0.50 & .05 & 0.05 & & 0.662 & -0.038 & -5.4 & & .495 & -0.04 & -8 & & 0.6 & -0 & 429 \\
\hline 0.70 & 0.50 & 0.05 & 0.10 & 0 & 0.646 & -0.054 & -7.7 & & 0.477 & -0.06 & -11. & & 0.646 & -0 . & -7.750 \\
\hline 0.70 & 0.50 & 10 & 0.00 & & 0.667 & -0.033 & -4.7 & & 0.500 & -0.0 & -7 & & 0.667 & -0 . & 714 \\
\hline 0.70 & 0.50 & 10 & 0.05 & 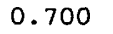 & 0.646 & -0.054 & -7.7 & & 0.477 & -0.06 & -11 & & 0.646 & -0 . & -7.750 \\
\hline 0.70 & 0.50 & 10 & 0.1 & & .628 & -0.072 & & & 0.458 & -0.08 & 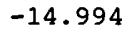 & & 0. & -0 . & -10.286 \\
\hline 0.70 & 0.70 & 0.00 & 0.00 & 0 & 0.700 & 0.000 & 0.0 & 0.6 & 0.620 & 0.00 & 0 . & & 0. & 0 . & 000 \\
\hline 0.70 & 0.70 & 0.00 & 0.05 & 0 & 0.668 & -0.032 & -4.5 & 0.620 & 0.585 & -0.03 & -5 & & 0.738 & -0 . & -3.556 \\
\hline 0.70 & 0.70 & 0.00 & 0.10 & & 0.642 & -0.0 & -8.3 & & 0.5 & -0.06 & -10 & & 0. & -0 & -6 \\
\hline 0.70 & 0.70 & 0.05 & 0.0 & & 0.695 & -0.0 & -0. & 0. & 0.615 & -0.0 & -0 . & & 0. & -0 & 53 \\
\hline 0.70 & 0.70 & 0.05 & 0.0 & & 2 & -0.0 & -5 & & 8 & -0 . & & & & -0 & \\
\hline 0.70 & 0.70 & 0.05 & 0.1 & & .634 & -0.06 & -9.4 & 0.6 & .548 & -0.07 & -11 . & & 0. & -0 . & -7.539 \\
\hline 0.70 & 0.70 & 0.10 & 0.0 & & 692 & -0.00 & -1 & & .612 & -0.0 & & & & & $-c$ \\
\hline 0.70 & 0.70 & 0.10 & 0.0 & & & -0.0 & $-\overline{6}$ & & .574 & -0.0 & -7 & & & -0 & -4 \\
\hline 0.70 & 0.70 & 0.10 & 0. & & 8 & -0.0 & $10+2$ & 0.6 & 2 & -0 . & $12+3$ & & & -0 & 12 \\
\hline 0.90 & 0.30 & 0.00 & 0.0 & & 0.5 & 0.0 & & & & 0. & & & & & \\
\hline 0.90 & 0.30 & 0.00 & 0.0 & & & -0 . & -2 & & & & & & & & \\
\hline 0.90 & 0.30 & 0.00 & 0. & & & -0.0 & -4 & & 1 & -0.0 & 10 & & & -0 & -6 \\
\hline 0.90 & 0.30 & 0.05 & 0. & & 5 & -0 . & -6 & & & -0 . & & & & & \\
\hline 0.90 & 0.30 & 0.05 & $0 . c$ & & & -0.0 & -8.4 & & 34 & -0.1 & -15 & & & & -12 \\
\hline 0.90 & 0.30 & 0.05 & 0. & & & -0. & & & & & & & & & \\
\hline 0.90 & 0.30 & 0.10 & 0.0 & & 797 & -0.1 & & & 10 & -0.1 & & & & -0 & -1 \\
\hline 0.90 & 0.30 & 0.10 & 0.0 & & & -0.1 & & & & -0.2 & & & & & \\
\hline 0.90 & 0.30 & 0.10 & 0 . & & & -0.1 & $15+2>$ & & .482 & -0.2 & . & & & -0 & $-2 z$ \\
\hline 0.90 & 0.50 & 0.00 & 0.0 & & & & & & & & & & & & \\
\hline & 0.50 & 0.00 & 0. & & & -0 . & -4 & & & -0 . & & & & -0 & -4.194 \\
\hline 0.90 & 0.50 & 0.00 & 0. & & & -0.0 & -7 & & & -0.1 & 10 & & & & -7.889 \\
\hline & 0.50 & 0.05 & & & & -0 . & -4 & & & & & & & & 194 \\
\hline 0.90 & 0.50 & 0.05 & & & & -0 & -8 & & & & & & & & \\
\hline & 0.50 & 0.05 & 0 . & & & -0 & & & & & & & & & -12 \\
\hline & 0.50 & 0.10 & & & & -0. & & & & -0.1 & & & & & -7.889 \\
\hline & 0.50 & 0.10 & 0. & & & -0.3 & & & & & & & & & \\
\hline & 0.50 & 0.10 & & & & -0 . & -16 & & .608 & -0.2 & -2 & & & -0 . & -16.000 \\
\hline & & 0.00 & 0. & & & 0. & & & & & & & & & \\
\hline & 0.70 & 0.00 & & & 5 & -0.0 & -6 & & .793 & -0.0 & & & & -0 . & -4.555 \\
\hline & 0.70 & 0.00 & 0 . & & & -0.1 & $11+2$ & & & & -15 & & & & \\
\hline & 0.70 & 0.05 & 0.8 & & 0.879 & -0.021 & 2.2 & 0.0 & 0.836 & -0.02 & -3. & 0.926 & 0.911 & -0. & -1.697 \\
\hline & & 0.05 & & & 0.824 & -0.0 & -8.4 & & 0.766 & $-0.0 s$ & -11 & & & & \\
\hline 0.90 & 0.70 & 0.05 & 0. & 0.900 & 0.7 & -0.125 & -13 & 0.863 & 0.707 & -0.156 & -18.103 & 0.926 & 0.828 & -0.098 & -10.607 \\
\hline & 0.70 & 0.10 & 0.0 & & 0.861 & -0.0 & -4.28 & & 0.813 & -0.050 & & & & & \\
\hline 0.90 & 0.70 & 0.10 & 0. & & 0.806 & -0.0 & -10 & & 0.7 & -0.119 & -13 & & 0.853 & -0 . & -7.937 \\
\hline & & & & & 0.756 & -0.144 & & 3 & 0.684 & 0.1 & & & & 4 & -12.285 \\
\hline
\end{tabular}

(SMC) Simple matching coefficient; $(J)$ Jaccard's coefficient; $(N L)$ Nei and Li's coefficient. $(s)$ Total similarity; $(p)$ proportion of similarity due to shared positive RAPD bands; $\left(f_{p}\right)$ probability of false positives; $\left(f_{n}\right)$ probability of false negatives.

the estimated value. Once the bias is known, it can be used to determine whether the RAPD protocol has been optimized sufficiently to provide accurate enough estimates of the similarities. With certain DNA samples, however, ad- equate optimization may not be possible. All is not lost, though, in these cases. A companion paper ${ }^{(30)}$ will discuss correcting for the bias in genetic similarity coefficients when estimates of the proportions of false positives and false neg- atives are available from replicate observations.

\section{ACKNOWLEDGMENTS}

This manuscript benefited from discus- 
sions with James McFerson and Muhammad Lodhi. Thanks go to Robert Bernatzky, Bruce Reisch, Ray Schnell, and an anonymous reviewer who read the manuscript critically and provided useful suggestions.

\section{REFERENCES}

1. Welsh, J. and M. McClelland. 1990. Fingerprinting genomes using PCR with arbitrary primers. Nucleic Acids Res. 18: 7213-7218.

2. Williams, J.G.K., A.R. Kubelik, K.J. Livak, J.A. Rafalski, and S.V. Tingey. 1990. DNA polymorphisms amplified by arbitrary primers are useful as genetic markers. $\mathrm{Nu}$ cleic Acids Res. 18: 6531-6535.

3. Reiter, R.S., J.G.K. Williams, K.A. Feldmann, J.A. Rafalski, S.V. Tingey, and P.A. Scolnik. 1992. Global and local genome mapping in Arabidopsis thaliana by using recombinant inbred lines and random amplified polymorphic DNAs. Proc. Natl. Acad. Sci. 89: 1477-1481.

4. Roy, A., N. Frascaria, J. MacKay, and J. Bousquet. 1992. Segregating random amplified polymorphic DNAs (RAPDs) in Betula alleghaniensis. Theor. Appl. Genet. 85: $173-180$.

5. Rieseberg, L.H., H. Choi, R. Chan, and C. Spore. 1993. Genomic map of a diploid hybrid species. Heredity 70: 285-293.

6. Torres, A.M., N.F. Weeden, and A. Martin. 1993. Linkage among isozyme, RFLP and RAPD markers in Vicia faba. Theor. Appl. Genet. 85: 937-945.

7. Stiles, J.I., C. Lemme, S. Sondur, M.B. Morshidi, and R. Manshardt. 1993. Using randomly amplified polymorphic DNA for evaluating genetic relationships among papaya cultivars. Theor. Appl. Genet. 85: 697-701.

8. Tibayrenc, M., K. Neubauer, C. Barnabé, F. Guerrini, D. Skarecky, and F.J. Ayala. 1993. Genetic characterization of six parasitic protozoa: Parity between randomprimer DNA typing and multilocus enzyme electrophoresis. Proc. Natl. Acad. Sci. 90: 1335-1339.

9. $\mathrm{Hu}, \mathrm{J}$. and C.F. Quiros. 1991. Identification of broccoli and cauliflower cultivars with RAPD markers. Plant Cell Rep. 10: 505-511.

10. Kresovich, S., J.G.K. Williams, J.R. McFerson, E.J. Routman, and B.A. Schaal. 1992. Characterization of genetic identities and relationships of Brassica oleracea L. via a random amplified polymorphic DNA assay. Theor. Appl. Genet. 85: 190-196.

11. Koller, B., A. Lehmann, J.M. McDermott, and C. Gessler. 1993. Identification of apple cultivars using RAPD markers. Theor. Appl. Genet. 85: 901-904.

12. Mulcahy, D.L., M. Cresti, S. Sansavini, G.C. Douglas, H.F. Linskens, G.B.
Mulcahy, R. Vignani, and M. Pancaldi. 1993. The use of random amplified polymorphic DNAs to fingerprint apple genotypes. Sci. Hortic. 54: 89-96.

13. Torres, A.M., T. Millán, and J.I. Cubero. 1993. Identifying rose cultivars using random amplified polymorphic DNA markers. HortScience 28: 333-334.

14. Yang, X. and C. Quiros. 1993. Identification and classification of celery cultivars with RAPD markers. Theor. Appl. Genet. 86: 205-212.

15. Kazan, K., J.M. Manners, and D.F. Cameron. 1993. Genetic relationships and variation in the Stylosanthes guianensis species complex assessed by random amplified polymorphic DNA. Genome 36: 43-49.

16. Kazan, K., J.M. Manners, and D.F. Cameron. 1993. Genetic variation in agronomically important species of Stylosanthes determined using random amplified polymorphic DNA markers. Theor. Appl. Genet. 85: 882-888.

17. Tinker, N.A., M.G. Fortin, and D.E Mather. 1993. Random amplified polymorphic DNA and pedigree relationships in spring barley. Theor. Appl. Genet. 85: 976-984.

18. Klein-Langhorst, R.M., A. Vermunt, R. Weide, T. Liharska, and P. Zabel. 1991. Isolation of molecular markers for tomato (L. esculentum) using random amplified polymorphic DNA (RAPD). Theor. Appl. Genet. 83: 108-114.

19. Heun, M. and T. Helentjaris. 1993. Inheritance of RAPDs in $\mathrm{F}_{1}$ hybrids of corn. Theor. Appl. Genet. 85: 961-968.

20. Riedy, M.F., W.J. Hamilton III, and C.F. Aquadro. 1992. Excess of non-parental bands in offspring from known primate pedigrees assayed using RAPD PCR. $\mathrm{Nu}$ cleic Acids Res. 20: 918.

21. Ellsworth, D.L., K.D. Rittenhouse, and R.L. Honeycutt. 1993. Artifactual variation in randomly amplified polymorphic DNA banding patterns. BioTechniques 14: 214-217.

22. Weeden, N.F., G.M. Timmerman, M. Hemmat, B.E. Kneen, and M.A. Lodhi. 1992. Inheritance and reliability of RAPD markers. In Applications of RAPD technology to plant breeding, Symposium Proceedings, pp. 12-17. Crop Science Society of America, Madison, WI

23. Carlson, J.E., L.K. Tulsieram, J.C. Glaubitz, V.W.K. Luk, C. Kauffeldt, and R. Rutledge. 1991. Segregation of random amplified DNA markers in $\mathrm{F}_{1}$ progeny of conifers. Theor. Appl. Genet. 83: 194-200.

24. Sneath, P.H.A. and R.R. Sokal. 1973. Numerical taxonomy. W.H. Freeman and Company, San Francisco, CA.

25. Jaccard, P. 1908. Nouvelles recherches sur la distribution florale. Bull. Soc. Vaud. Sci. Nat. 44: 223-270.

26. Vierling, R.A. and H.T. Nguyen. 1992. Use of RAPD markers to determine the genetic diversity of diploid wheat genotypes. Theor. Appl. Genet. 84: 835-838.

27. Nei, M. and W.-H. Li. 1979. Mathematical model for studying genetic variation in terms of restriction endonucleases. Proc. Natl. Acad. Sci. 76: 5269-5273.

28. Chalmers, K.J., R. Waugh, J.I. Sprent, A.J. Simons, and W. Powell. 1992. Detection of genetic variation between and within populations of Gliricidia sepium and $G$. maculata using RAPD markers. Heredity 69: 465-472.

29. Lashermes, P., J. Cros, P. Marmey, and A. Charrier. 1993. Use of random amplified DNA markers to analyse genetic variability and relationships of Coffea species. Genet. Resources Crop Evol. 40: 91-99.

30. Lamboy, W.F. 1994. Computing genetic similarity coefficients from RAPD data: Correcting for the effects of PCR artifacts caused by variation in experimental conditions. PCR Methods Applic. (this issue)

Received April 15, 1994; accepted in revised form June 15, 1994. 


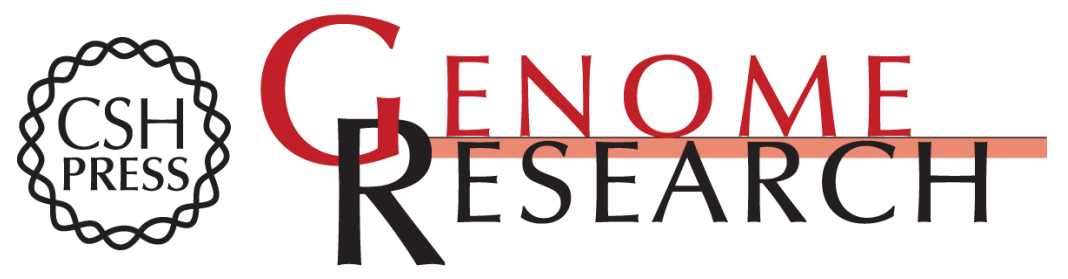

\section{Computing genetic similarity coefficients from RAPD data: the effects of PCR artifacts.}

W F Lamboy

Genome Res. 1994 4: 31-37

References This article cites 27 articles, 3 of which can be accessed free at:

http://genome.cshlp.org/content/4/1/31.full.html\#ref-list-1

License

Email Alerting Receive free email alerts when new articles cite this article - sign up in the box at the Service top right corner of the article or click here.

\section{Affordable, Accurate} Sequencing.

To subscribe to Genome Research go to:

https://genome.cshlp.org/subscriptions 